\title{
First report of field evolved resistance to agrochemicals in dengue mosquito, Aedes albopictus (Diptera: Culicidae), from Pakistan
}

\author{
Hafiz Azhar Ali Khan ${ }^{1 *}$, Waseem Akram²*, Khurram Shehzad ${ }^{3}$ and Essam A Shaalan ${ }^{4}$
}

\begin{abstract}
Background: Agrochemicals have been widely used in Pakistan for several years. This exposes mosquito populations, particularly those present around agricultural settings, to an intense selection pressure for insecticide resistance. The aim of the present study was to investigate the toxicity of representative agrochemicals against various populations of Aedes albopictus (Skuse) collected from three different regions from 2008-2010.

Results: For organophosphates and pyrethroids, the resistance ratios compared with susceptible Lab-PK were in the range of 157-266 fold for chlorpyrifos, 24-52 fold for profenofos, 41-71 fold for triazofos, and 15-26 fold for cypermethrin, 15-53 fold for deltamethrin and 21-58 fold for lambdacyhalothrin. The resistance ratios for carbamates and new insecticides were in the range of 13-22 fold for methomyl, 24-30 fold for thiodicarb, and 41101 fold for indoxacarb, 14-27 fold for emamectin benzoate and 23-50 fold for spinosad. Pair wise comparisons of the log $L C_{50}$ of insecticides revealed correlation among several insecticides, suggesting a possible cross resistance mechanism. Moreover, resistance remained stable across 3 years, suggesting field selection for general fitness had also taken place for various populations of Ae. albopictus.
\end{abstract}

Conclusion: Moderate to high level of resistance to agrochemicals in Pakistani field populations of Ae. albopictus is reported here first time. The geographic extent of resistance is unknown but, if widespread, may lead to problems in future vector control.

\section{Background}

Dengue fever and dengue hemorrhagic fever (DF/DHF) are vector borne diseases of public health concerns in tropical and subtropical parts of the world [1], affecting millions of people annually [2]. The incidence of DF and DHF has increased cyclically in Pakistan since the first recognized outbreak in 1994 with Ae. albopictus (Skuse) as the core mosquito vector in this respect [3]. Currently, controlling this vector with insecticidal habitat spraying remains an important option to minimize the incidence of dengue fever [4], resulting in resurgence and development of insecticidal resistance.

\footnotetext{
* Correspondence: azhar_naturalist@yahoo.com; areeba14@yahoo.com 'Department of Entomology, University College of Agriculture, Bahauddin Zakariya University, Multan, Pakistan

${ }^{2}$ Department of Agri-Entomology, University of Agriculture, Faisalabad, Pakistan

Full list of author information is available at the end of the article
}

Insecticide resistance has become a limiting factor in the use of these compounds in chemical control of many insect pests. The exploration of more efficient toxic chemicals and other control tactics are necessary with the increasing world population and preservation of species diversity [5]. Frequent use of chemicals, such as pesticides, coupled with monoculture crops on a large scale, has generated pesticide resistance in insect pests, resurgence and difficulties in pest management [6]. By 2007, intensive use of pesticides had resulted in at least 553 arthropod species resistant to one or more classes of insecticides (organochlorines, organophosphates, carbamates and pyrethroids) [7]. Of these, 60 percent are agricultural pests and the remaining 40 percent are pests of medical importance [8]. Resistance in medical pests or disease vectors is a serious threat to the control of vector-borne diseases, owing to the fact of insecticide-based strategies such as insecticide treated nets, indoor residual spraying, insecticide treatment of

\section{Biomed Central}


breeding habitats and also because of agricultural practices [9].

Various disease vectors are present in agro-ecozones and are therefore likely to be exposed to chemicals used to control agricultural pests. Despite the lack of concrete evidence, the massive use of agrochemicals has been considered as a key factor contributing to the emergence of vector resistance to insecticides [10]. Insecticide resistance in disease vectors due to the selection pressure from agrochemicals has been reported from different parts of the world [9,11-15], however, no such reports have so far been reported from Pakistan.

Crop losses caused by insect pest in Pakistan are upto $56 \%$, and $20-40 \%$ of these losses are in cotton, Gossypium hirsutum L. [16]. As a result, agrochemicals with broad toxicity to target pests and non target organisms are being widely used in cotton insect pest management. The overuse of chemicals can lead to the phenomenon of insecticide resistance both in target and non target organisms. In the current study, we were interested to establish whether Ae. albopictus, present in cotton cultivated fields, had developed resistance to agrochemicals (organophosphates, carbamates, pyrethroids and newer compounds). These chemicals are commonly used for the control of cotton insect pests in Punjab province, Pakistan [17]. We were also interested in investigating whether resistance to different insecticides was increasing or remained the same from 2008-2010. The present paper reports the first known occurrence of high level resistance to agrochemicals in $A e$. albopictus. The data from such studies are expected to help in future management strategies so that the development of resistance is delayed to a maximum in Ae. albopictus under field conditions of Pakistan.

\section{Materials and methods \\ Mosquitoes}

We collected natural populations of Ae. albopictus from upper Punjab, Pakistan (Lahore, Sargodha and Faisalabad districts) from 2008-2010. The growers usually undertake more insecticides on cotton than any other crop [17]. We therefore collected Ae. albopictus populations from cotton fields as there were higher chances of evolution of resistance on cotton than other crops. The collection sites within the districts were kept constant across three years. Moreover, a group of Ae. Albopictus collected in a date from a determinate place was considered as a population. The samples of larvae and pupae from each district were colonized under laboratory conditions at $27 \pm 1^{\circ} \mathrm{C}$ and $65-70 \% \mathrm{RH}$. Larvae were fed on fish food $\left(\right.$ TetraMin $\left.{ }^{\circledR}\right)$. Adults were kept in plastic cages $(30 \times 40 \times 40)$ where males were provided cotton wicks soaked with $20 \%$ sucrose solution and females were fed on blood of white rats thrice a week [4]. Fourth instar larvae of the F1 progeny were reared for bioassays. However, some bioassays were performed on the F2 generation due to insufficient numbers of F1 progeny. The laboratory susceptible strain of Ae. albopictus was collected in 2005 from mountainous areas of Islamabad with zero or very low chemical use and it was designated as Lab-PK. This population was reared in the laboratory for $>40$ generations without exposure to insecticides. The Lab-PK population showed lowest $\mathrm{LC}_{50}$ values for all the tested insecticides, and hence was used as a reference strain to calculate resistance ratios.

\section{Insecticides}

Commercial formulations of different insecticides used for bioassays consisted of chlorpyrifos (Lorsban $40 \mathrm{EC}$, Dow Agro Sciences, United Kingdom), profenofos (Curacron 50 EC, Syngenta Crop Protection, Switzerland), triazofos (Hostathion 40 EC, Bayer Crop Science), cypermethrin (Arrivo 10 EC, FMC, Philadelphia; PA), deltamethrin (Decis Super 10.5 EC, Bayer Crop Science, Multan, Pakistan), lambdacyhalothrin (Karate EC Syngenta Crop Protection Switzerland ), methomyl (Lannate LV 239 g [AI]/liter, DuPont, Pakistan), thiodicarb (Larvin SC 375 g [AI]/liter, Bayer Crop Science, Multan, Pakistan), indoxacarb (Steward 15SC, DuPont, Pakistan), spinosad (Tracer 24SC, Dow Agro Sciences, UK) and emamectin benzoate (Proclaim 1.9 EC, Syngenta, UK)

\section{Bioassays}

Bioassays were performed as described previously [18] using acetone solution of insecticides. One milliliter of appropriate insecticide solution was dispensed with a pipette above the water surface in each glass beaker containing $99 \mathrm{ml}$ of distilled water. Each insecticide was tested within a range of seven to eight concentrations to determine $\mathrm{LC}_{50}$ value, including controls, and each concentration was replicated at least four times. Ten $4^{\text {th }}$ instar larvae were placed in the glass beaker in each replication and the total number of larvae tested per concentration was 40 . The bioassays were kept at a temperature of $27 \pm 1^{\circ} \mathrm{C}, 65 \% \mathrm{RH}$ and a photoperiod of $14 \mathrm{~L}$ : 10D hours. Mortality was recorded after 24 hours [18], except for spinosad, which was assayed after 48 hours due to the slower acting nature of this insecticide. Larvae were considered dead if they could not be induced to move when probed with a probe.

\section{Stability of resistance}

A decline or increase in resistance to the tested insecticides in field populations from 1 year to the next was measured by calculating $R$ values i.e., respond per month. The $\mathrm{R}$ values were estimated as below:

$$
\mathrm{R}=\left[\log \left(\text { final } \mathrm{LC}_{50}\right)-\log \left(\text { initial } \mathrm{LC}_{50}\right)\right] / n,
$$


Where ' $n$ ' is the number of months (6 months) after which a second population was collected from the same field. Decline or increase in resistance is presented in and/or + values of $R$ [16].

\section{Data analysis}

Mortality data, where necessary, were corrected by Abbott's formula [19]. Data were analyzed using probit analysis based on Finney (1971) [20] to determine the $\mathrm{LC}_{50}$ values and their 95\% fiducial limits (FLs) using MINITAB 15 statistical software [21]. Due to the inherent variability of bioassays, pair wise comparisons of $\mathrm{LC}_{50}$ values were made, and if $95 \%$ FLs of two treatments did not overlap at $1 \%$ level of significance, they were considered significant [22]. Resistance ratios (RRs) were calculated by dividing the $\mathrm{LC}_{50}$ values of field populations with $\mathrm{LC}_{50}$ of susceptible Lab-PK. To determine cross resistance among the tested insecticides, pair wise correlation coefficients $(r)$ of $\log \mathrm{LC}_{50}$ values of the field populations were also calculated. The slopes of regression lines were compared using $t$-test in Statistix 8.1 [23].

To determine insecticide resistance, the level of insecticide resistance was scaled by using resistance ratios (RRs) in terms of widely accepted values as follows: susceptibility $(R R=1)$, low resistance $(R R=2-10)$, moderate resistance $(R R=11-30)$, high resistance $(R R=31$ $100)$ and a very high resistance $(R R>100)$ [24].

\section{Results}

\section{Toxicity of insecticides to susceptible population}

The results obtained from the bioassays with the LabPK population (Table 1,2) revealed that chlorpyrifos was significantly more toxic (non overlapping of $95 \%$ FL; $\mathrm{P}<0.01)$ than the insecticides tested viz., profenofos, triazofos, cypermethrin, deltamethrin, lambdacyhalothrin, methomyl, thiodicarb, indoxacarb, emamectin benzoate and spinosad. Emamectin benzoate was the least effective compound than the other insecticides tested. The slopes of regression lines of all the insecticides were similar $(\mathrm{P}>0.05)$.

\section{Toxicity of insecticides to field population}

The toxicity of all eleven insecticides against field population was significantly lower than Lab-PK (95\% FL did not overlap, Table 1, 2).

\section{Organophosphates}

The levels of resistance to chlorpyrifos in samples from all the three districts of Punjab were generally very high, with resistance ratios 157-266 fold. All the field populations tested with chlorpyrifos in 3 consecutive years showed very high levels of resistance $(R R>100)$. The highest level of resistance (266 fold) was observed in
March 2009 from Faisalabad, whereas the lowest (157 fold) was from Lahore in March 2009 (Table 1). The slopes of regression lines of all the populations were significantly shallower than Lab-PK population $(\mathrm{P}<0.05)$.

Among 15 populations tested for profenofos, five populations had moderate levels of resistance (24-26 fold) than the Lab-PK population, and the remaining 10 populations had high levels of resistance (34-52 fold). The highest level of resistance was found in population from Faisalabad in March 2010, whereas the lowest level was found in population from Sargodha in September 2008 (Table 1). The slopes of regression lines of all the populations were significantly shallower than the LabPK population $(\mathrm{P}<0.05)$.

All the 15 populations tested for triazofos had high levels of resistance (41-71 fold). The highest level of resistance was seen in populations from Sargodha in September 2009, whereas the lowest level was observed in populations from Lahore in September 2009 (Table 1).

\section{Pyrethroids}

Moderate levels of resistance was found in all the populations tested against cypermethrin (15-26 fold, Table 1) compared with the lab-PK population. The lowest level of resistance was observed in populations from Sargodha in March 2010. The slopes of regression lines of all the populations were significantly shallower than Lab-PK population $(\mathrm{P}<0.05)$.

Moderate to high levels of resistance were observed in populations tested for deltamethrin (15- to 53 fold, Table 1). One population from Lahore and four populations from Sargodha had moderate levels of resistance (15-25 fold) while the remaining populations were highly resistant (31-53 fold). Of 15 populations tested against lambdacyhalothrin, only three populations from Sargodha had moderate levels of resistance with resistance ratios ranging from 21-30 fold compared with Lab-PK population (Table 1). The slopes of regression lines of all the field populations were similar $(\mathrm{P}>0.05)$.

\section{Carbamates}

Methomyl was significantly less toxic to field populations $(\mathrm{P}<0.01)$ compared to Lab-PK. All the field populations tested for methomyl had moderate levels of resistance (13-22 fold) compared with Lab-PK (Table 2). The lowest level was observed in populations from Sargodha in September 2009. The slopes of regression lines of all the field populations were similar $(\mathrm{P}>0.05)$ but shallower than the Lab-PK $(\mathrm{P}<0.05)$.

Out of 15 populations tested for thiodicarb, three populations from Lahore, two from Faisalabad and three from Sargodha were moderately resistant with resistance ratios ranging from 24-30 fold compared with Lab-PK 
Table 1 Toxicity of organophosphates and pyrethroids against field populations of Ae. albopictus

\begin{tabular}{|c|c|c|c|c|c|c|c|c|c|c|}
\hline Insecticide & Location & Time & $\mathrm{LC}_{50}(95 \% \mathrm{FL})\left(\mu \mathrm{g} \mathrm{mL}^{-1}\right)$ & Slope \pm SE & $\chi^{2}$ & df & $\mathbf{P}$ & $\mathrm{RR}^{*}$ & $\mathrm{DR}^{* *}$ & $\mathrm{n}^{* * *}$ \\
\hline \multirow[t]{15}{*}{ Chlorpyrifos } & Lab-PK & & $0.009(0.002-0.013)$ & $2.25 \pm 0.31$ & 0.69 & 5 & 0.98 & 1 & - & 280 \\
\hline & Lahore & Mar. 2009 & $1.92(1.27-4.95)$ & $1.04 \pm 0.25$ & 5.73 & 6 & 0.46 & 156.6 & - & 320 \\
\hline & & Sep. 2009 & $2.61(1.35-13.21)$ & $0.59 \pm 0.26$ & 5.84 & 6 & 0.44 & 247.8 & 0.222 & 320 \\
\hline & & Mar. 2010 & $2.88(1.27-15.44)$ & $0.56 \pm 0.25$ & 3.95 & 6 & 0.68 & 242.2 & 0.030 & 320 \\
\hline & & Sep. 2010 & $3.36(1.33-8.55)$ & $0.67 \pm 0.26$ & 2.65 & 6 & 0.85 & 240 & 0.041 & 320 \\
\hline & Faisalabad & Sep. 2008 & $2.08(1.26-7.31)$ & $0.66 \pm 0.30$ & 3.34 & 6 & 0.77 & 224.4 & - & 320 \\
\hline & & Mar. 2009 & $2.40(1.39-18.57)$ & $0.59 \pm 0.27$ & 5.51 & 6 & 0.48 & 266 & 0.010 & 320 \\
\hline & & Sep. 2009 & $1.71(1.19-3.48)$ & $0.93 \pm 0.27$ & 5.76 & 6 & 0.45 & 190 & -0.014 & 320 \\
\hline & & Mar. 2010 & $2.00(1.32-5.15)$ & $0.84 \pm 0.28$ & 6.11 & 6 & 0.41 & 222 & -0.003 & 320 \\
\hline & & Sep. 2010 & $2.12(1.37-6.32)$ & $0.81 \pm 0.29$ & 6.21 & 6 & 0.40 & 235.6 & 0.001 & 320 \\
\hline & Sargodha & Sep. 2008 & $1.74(1.15-4.29)$ & $0.77 \pm 0.25$ & 6.51 & 6 & 0.37 & 193 & - & 320 \\
\hline & & Mar. 2009 & $1.89(1.25-4.77)$ & $0.81 \pm 0.27$ & 9.40 & 6 & 0.15 & 210 & 0.006 & 320 \\
\hline & & Sep. 2009 & $1.57(1.15-2.76)$ & $1.09 \pm 0.27$ & 11.3 & 6 & 0.08 & 174 & -0.007 & 320 \\
\hline & & Mar. 2010 & $1.48(1.16-2.23)$ & $1.57 \pm 0.35$ & 9.45 & 6 & 0.15 & 164 & -0.012 & 320 \\
\hline & & Sep. 2010 & $1.69(1.24-3.00)$ & $1.22 \pm 0.32$ & 10.3 & 6 & 0.11 & 187.8 & -0.002 & 320 \\
\hline \multirow[t]{16}{*}{ Profenofos } & Lab-PK & & $0.02(0.015-0.04)$ & $2.41 \pm 0.27$ & 2.44 & 6 & 0.88 & 1 & - & 320 \\
\hline & Lahore & Sep. 2008 & $0.56(0.43-0.77)$ & $1.25 \pm 0.22$ & 9.34 & 6 & 0.16 & 28 & - & 320 \\
\hline & & Mar. 2009 & $0.70(0.53-1.04)$ & $1.09 \pm 0.31$ & 5.24 & 5 & 0.39 & 35 & 0.016 & 280 \\
\hline & & Sep. 2009 & $0.73(0.55-1.07)$ & $1.10 \pm 0.40$ & 7.49 & 6 & 0.28 & 36.5 & 0.019 & 320 \\
\hline & & Mar. 2010 & $0.79(0.59-1.23)$ & $1.05 \pm 0.23$ & 5.90 & 6 & 0.43 & 39.5 & 0.025 & 320 \\
\hline & & Sep. 2010 & $0.82(0.52-2.26)$ & $0.62 \pm 0.23$ & 0.53 & 5 & 0.99 & 41 & 0.028 & 280 \\
\hline & Faisalabad & Sep. 2008 & $0.80(0.62-1.15)$ & $1.17 \pm 0.33$ & 11.6 & 6 & 0.07 & 40 & - & 320 \\
\hline & & Mar. 2009 & $0.82(0.64-1.18)$ & $1.19 \pm 0.24$ & 3.90 & 6 & 0.69 & 41 & 0.001 & 320 \\
\hline & & Sep. 2009 & $0.98(0.75-1.48)$ & $1.11 \pm 0.40$ & 7.38 & 6 & 0.29 & 49 & 0.015 & 320 \\
\hline & & Mar. 2010 & $1.03(0.77-1.62)$ & $1.03 \pm 0.37$ & 8.62 & 6 & 0.17 & 51.5 & 0.018 & 320 \\
\hline & & Sep. 2010 & $0.71(0.56-0.96)$ & $1.31 \pm 0.23$ & 9.78 & 6 & 0.13 & 35.5 & -0.009 & 320 \\
\hline & Sargodha & Sep. 2008 & $0.49(0.35-0.72)$ & $0.97 \pm 0.20$ & 1.95 & 5 & 0.86 & 24 & - & 280 \\
\hline & & Mar. 2009 & $0.54(0.42-0.73)$ & $1.44 \pm 0.23$ & 9.09 & 6 & 0.17 & 27 & 0.007 & 320 \\
\hline & & Sep. 2009 & $0.68(0.52-0.94)$ & $1.23 \pm 0.32$ & 6.44 & 6 & 0.38 & 34 & 0.024 & 320 \\
\hline & & Mar. 2010 & $0.52(0.35-0.84)$ & $0.91 \pm 0.25$ & 2.08 & 5 & 0.84 & 26 & 0.004 & 280 \\
\hline & & Sep. 2010 & $0.55(0.39-0.74)$ & $1.23 \pm 0.25$ & 8.09 & 6 & 0.17 & 27.5 & 0.008 & 320 \\
\hline \multirow[t]{15}{*}{ Triazofos } & Lab-PK & & $0.036(0.02-0.06)$ & $2.39 \pm 1.29$ & 8.06 & 6 & 0.23 & 1 & - & 320 \\
\hline & Lahore & Sep. 2008 & $1.80(1.18-4.71)$ & $0.75 \pm 0.20$ & 8.18 & 6 & 0.22 & 50 & - & 320 \\
\hline & & Mar. 2009 & $1.94(1.28-4.99)$ & $0.82 \pm 0.19$ & 11.8 & 6 & 0.07 & 53.9 & 0.005 & 320 \\
\hline & & Sep. 2009 & $1.49(1.11-2.44)$ & $1.16 \pm 0.27$ & 7.82 & 6 & 0.25 & 41.4 & -0.014 & 320 \\
\hline & & Mar. 2010 & $1.62(1.22-2.70)$ & $1.36 \pm 0.33$ & 8.20 & 6 & 0.23 & 45 & -0.008 & 320 \\
\hline & & Sep. 2010 & $1.80(1.28-3.50)$ & $1.12 \pm 0.31$ & 7.57 & 6 & 0.27 & 50 & 0 & 320 \\
\hline & Faisalabad & Sep. 2008 & $2.26(1.31-12.26)$ & $0.58 \pm 0.16$ & 5.89 & 5 & 0.32 & 62.8 & - & 280 \\
\hline & & Mar. 2009 & $2.00(1.29-6.08)$ & $0.79 \pm 0.29$ & 10.0 & 5 & 0.08 & 55.6 & -0.009 & 280 \\
\hline & & Sep. 2009 & $2.26(1.38-11.23)$ & $0.70 \pm 0.29$ & 6.29 & 5 & 0.28 & 62.8 & 0 & 280 \\
\hline & & Mar. 2010 & $2.26(1.42-8.69)$ & $0.81 \pm 0.32$ & 4.33 & 5 & 0.50 & 62.8 & 0 & 280 \\
\hline & Sargodha & Sep. 2008 & $2.57(0.55-4.51)$ & $0.64 \pm 0.29$ & 4.73 & 6 & 0.58 & 71.4 & - & 320 \\
\hline & & Mar. 2009 & $1.96(0.88-3.01)$ & $0.81 \pm 0.27$ & 8.49 & 6 & 0.21 & 54.5 & -0.019 & 320 \\
\hline & & Sep. 2009 & $1.72(0.99-2.50)$ & $1.04 \pm 0.29$ & 11.3 & 6 & 0.08 & 47.8 & -0.029 & 320 \\
\hline & & Mar. 2010 & $1.74(0.92-2.61)$ & $0.86 \pm 0.40$ & 5.63 & 6 & 0.47 & 48.3 & -0.028 & 320 \\
\hline & & Sep. 2010 & $2.18(0.89-3.47)$ & $0.87 \pm 0.31$ & 3.01 & 6 & 0.81 & 60.6 & -0.012 & 320 \\
\hline \multirow[t]{5}{*}{ Cypermethrin } & Lab-PK & & $0.04(0.02-0.09)$ & $2.41 \pm 0.45$ & 5.96 & 6 & 0.43 & 1 & - & 320 \\
\hline & Lahore & Sep. 2008 & $0.86(0.66-1.29)$ & $1.11 \pm 0.23$ & 8.62 & 6 & 0.20 & 21.5 & - & 320 \\
\hline & & Mar. 2009 & $0.71(0.57-0.95)$ & $1.40 \pm 0.35$ & 11.6 & 6 & 0.72 & 17.8 & -0.014 & 320 \\
\hline & & Sep. 2009 & $0.63(0.52-0.85)$ & $1.46 \pm 0.22$ & 10.4 & 6 & 0.11 & 15.8 & -0.023 & 320 \\
\hline & & Mar. 2010 & $0.89(0.69-1.30)$ & $1.16 \pm 0.24$ & 11.3 & 6 & 0.08 & 22.3 & 0.003 & 320 \\
\hline
\end{tabular}


Table 1 Toxicity of organophosphates and pyrethroids against field populations of Ae. albopictus (Continued)

\begin{tabular}{|c|c|c|c|c|c|c|c|c|c|c|}
\hline & & Sep. 2010 & $0.83(0.64-1.15)$ & $1.24 \pm 0.42$ & 6.43 & 6 & 0.38 & 20.8 & -0.003 & 320 \\
\hline & \multirow[t]{5}{*}{ Faisalabad } & Sep. 2008 & $1.02(0.78-1.54)$ & $1.11 \pm 0.37$ & 9.79 & 6 & 0.13 & 25.5 & - & 320 \\
\hline & & Mar. 2009 & $0.78(0.55-1.00)$ & $1.19 \pm 0.43$ & 6.56 & 6 & 0.36 & 19.5 & -0.019 & 320 \\
\hline & & Sep. 2009 & $0.94(0.54-1.34)$ & $0.82 \pm 0.22$ & 4.21 & 6 & 0.65 & 23.5 & -0.006 & 320 \\
\hline & & Mar. 2010 & $0.94(0.60-1.27)$ & $1.00 \pm 0.30$ & 6.29 & 6 & 0.39 & 23.5 & -0.006 & 320 \\
\hline & & Sep. 2010 & $0.87(0.53-1.21)$ & $0.87 \pm 0.23$ & 4.03 & 5 & 0.55 & 21.8 & -0.012 & 280 \\
\hline & \multirow[t]{5}{*}{ Sargodha } & Sep. 2008 & $0.68(0.52-0.83)$ & $1.49 \pm 0.41$ & 9.15 & 6 & 0.17 & 17 & - & 320 \\
\hline & & Mar. 2009 & $0.58(0.46-0.70)$ & $1.55 \pm 0.24$ & 11.9 & 6 & 0.06 & 14.5 & -0.012 & 320 \\
\hline & & Sep. 2009 & $0.69(0.50-0.87)$ & $1.26 \pm 0.44$ & 7.49 & 6 & 0.28 & 17.3 & 0.001 & 320 \\
\hline & & Mar. 2010 & $0.70(0.55-0.86)$ & $1.57 \pm 0.52$ & 8.37 & 6 & 0.21 & 17.5 & 0.002 & 320 \\
\hline & & Sep. 2010 & $0.66(0.49-0.83)$ & $1.35 \pm 0.37$ & 10.5 & 6 & 0.11 & 16.5 & -0.002 & 320 \\
\hline \multirow[t]{16}{*}{ Deltamethrin } & Lab-PK & & $0.028(0.02-0.04)$ & $2.29 \pm 0.24$ & 3.39 & 6 & 0.76 & 1 & - & 320 \\
\hline & \multirow[t]{5}{*}{ Lahore } & Sep. 2008 & $1.06(0.74-1.34)$ & $1.18 \pm 0.18$ & 4.08 & 6 & 0.67 & 37.9 & - & 320 \\
\hline & & Mar. 2009 & $1.22(0.76-1.68)$ & $0.98 \pm 0.25$ & 4.34 & 6 & 0.63 & 43.6 & 0.010 & 320 \\
\hline & & Sep. 2009 & $0.92(0.61-1.23)$ & $1.02 \pm 0.23$ & 4.49 & 6 & 0.61 & 32.9 & -0.010 & 320 \\
\hline & & Mar. 2010 & $0.41(0.262-5.61)$ & $0.82 \pm 0.13$ & 0.55 & 4 & 0.97 & 14.6 & -0.069 & 240 \\
\hline & & Sep. 2010 & $1.15(0.57-1.74)$ & $0.72 \pm 0.16$ & 2.25 & 5 & 0.81 & 41.1 & 0.006 & 280 \\
\hline & \multirow[t]{5}{*}{ Faisalabad } & Sep. 2008 & $1.48(0.82-2.14)$ & $0.88 \pm 0.21$ & 4.12 & 6 & 0.66 & 52.8 & - & 320 \\
\hline & & Mar. 2009 & $1.35(0.77-1.92)$ & $0.92 \pm 0.36$ & 1.57 & 5 & 0.91 & 48.2 & -0.007 & 280 \\
\hline & & Sep. 2009 & $1.16(0.72-1.60)$ & $0.95 \pm 0.21$ & 3.78 & 6 & 0.71 & 41.4 & -0.018 & 320 \\
\hline & & Mar. 2010 & $1.27(0.70-1.83)$ & $0.82 \pm 0.40$ & 3.81 & 6 & 0.70 & 45.4 & -0.011 & 320 \\
\hline & & Sep. 2010 & $1.31(0.77-1.71)$ & $1.04 \pm 0.38$ & 8.61 & 6 & 0.17 & 46.8 & -0.009 & 320 \\
\hline & \multirow[t]{5}{*}{ Sargodha } & Sep. 2008 & $0.70(0.51-0.88)$ & $1.33 \pm 0.52$ & 6.10 & 6 & 0.41 & 25 & - & 320 \\
\hline & & Mar. 2009 & $0.88(0.63-1.13)$ & $1.21 \pm 0.28$ & 5.52 & 6 & 0.48 & 31.4 & 0.017 & 320 \\
\hline & & Sep. 2009 & $0.63(0.49-0.77)$ & $1.66 \pm 0.34$ & 11.9 & 6 & 0.06 & 22.5 & -0.008 & 320 \\
\hline & & Mar. 2010 & $0.68(0.44-0.99)$ & $0.99 \pm 0.42$ & 5.65 & 6 & 0.46 & 24.3 & -0.002 & 320 \\
\hline & & Sep. 2010 & $0.68(0.51-0.83)$ & $1.44 \pm 0.36$ & 9.20 & 6 & 0.16 & 24.3 & -0.002 & 320 \\
\hline \multirow[t]{16}{*}{ Lambdacyh-alothrin } & Lab-PK & & $0.02(0.014-0.033)$ & $2.32 \pm 0.51$ & 3.56 & 6 & 0.74 & 1 & - & 320 \\
\hline & \multirow[t]{5}{*}{ Lahore } & Sep. 2008 & $0.91(0.59-1.22)$ & $1.00 \pm 0.16$ & 9.41 & 5 & 0.09 & 45.5 & - & 280 \\
\hline & & Mar. 2009 & $1.16(0.72-1.60)$ & $0.97 \pm 0.24$ & 8.61 & 5 & 0.13 & 58 & 0.018 & 280 \\
\hline & & Sep. 2009 & $0.91(0.61-1.20)$ & $1.10 \pm 0.26$ & 9.22 & 5 & 0.10 & 45.5 & 0 & 280 \\
\hline & & Mar. 2010 & $0.90(0.56-1.23)$ & $0.92 \pm 0.28$ & 8.24 & 5 & 0.14 & 45 & -0.001 & 280 \\
\hline & & Sep. 2010 & $1.15(0.63-1.66)$ & $0.81 \pm 0.18$ & 6.58 & 5 & 0.25 & 57.5 & 0.017 & 280 \\
\hline & \multirow[t]{5}{*}{ Faisalabad } & Sep. 2008 & $0.84(0.57-1.11)$ & $1.05 \pm 0.20$ & 9.28 & 6 & 0.16 & 42 & - & 320 \\
\hline & & Mar. 2009 & $0.65(0.44-0.87)$ & $1.10 \pm 0.31$ & 9.71 & 6 & 0.14 & 33 & -0.018 & 320 \\
\hline & & Sep. 2009 & $0.83(0.61-1.05)$ & $1.28 \pm 0.44$ & 12.0 & 6 & 0.06 & 41.5 & -0.001 & 320 \\
\hline & & Mar. 2010 & $0.81(0.59-1.03)$ & $1.27 \pm 0.36$ & 8.85 & 6 & 0.18 & 40.5 & -0.003 & 320 \\
\hline & & Sep. 2010 & $0.78(0.52-1.04)$ & $1.03 \pm 0.26$ & 8.41 & 6 & 0.21 & 39 & -0.005 & 320 \\
\hline & \multirow[t]{5}{*}{ Sargodha } & Sep. 2008 & $0.42(0.30-0.54)$ & $1.42 \pm 0.61$ & 10.7 & 6 & 0.09 & 21 & - & 320 \\
\hline & & Mar. 2009 & $0.59(0.46-0.72)$ & $1.62 \pm 0.46$ & 10.1 & 6 & 0.12 & 29.5 & 0.025 & 320 \\
\hline & & Sep. 2009 & $0.64(0.49-0.80)$ & $1.56 \pm 0.44$ & 10.4 & 6 & 0.11 & 32 & 0.030 & 320 \\
\hline & & Mar. 2010 & $0.55(0.43-0.68)$ & $1.56 \pm 0.48$ & 8.10 & 6 & 0.23 & 27.5 & 0.020 & 320 \\
\hline & & Sep. 2010 & $0.65(0.45-0.86)$ & $1.09 \pm 0.26$ & 7.05 & 6 & 0.32 & 32.5 & 0.032 & 320 \\
\hline
\end{tabular}

*Resistance ratio $=\mathrm{LC}_{50}$ field population $/ \mathrm{LC}_{50}$ of susceptible strain

** Rate of increase or decrease in resistance, ${ }^{* * *}$ Number of larvae tested in a bioassay

(Table 2). The remaining 7 populations were highly resistant to this chemical (31-37 fold).

\section{New insecticides}

Among the 15 populations tested with indoxacarb, only one population from Faisalabad in September 2010 showed very high resistance (101 fold) while the remaining populations were highly resistant with resistance ratios ranging from 41-89 fold compared with Lab-PK (Table 2). The lowest level of resistance was found in populations from Lahore in September 2008. All the populations tested for emamectin benzoate were 
Table 2 Toxicity of carbamates and new insecticides against field populations of Ae. albopictus

\begin{tabular}{|c|c|c|c|c|c|c|c|c|c|c|}
\hline Insecticide & Location & Time & $\mathrm{LC}_{50}(95 \% \mathrm{FL})\left(\mu \mathrm{g} \mathrm{mL}^{-1}\right)$ & Slope \pm SE & $\chi^{2}$ & df & $\mathbf{P}$ & $\mathrm{RR}^{*}$ & $\mathrm{DR}^{* *}$ & $\mathrm{n}^{* * *}$ \\
\hline \multirow[t]{16}{*}{ Methomyl } & Lab-PK & & $0.06(0.03-0.17)$ & $2.56 \pm 033$ & 9.99 & 6 & 0.12 & 1 & - & 320 \\
\hline & Lahore & Sep. 2008 & $1.31(0.71-1.91)$ & $0.81 \pm 0.40$ & 3.13 & 6 & 0.79 & 21.8 & _- & 320 \\
\hline & & Mar. 2009 & $1.11(0.63-1.51)$ & $0.84 \pm 0.26$ & 4.61 & 6 & 0.59 & 18.5 & -0.012 & 320 \\
\hline & & Sep. 2009 & $0.95(0.66-1.24)$ & $1.14 \pm 0.23$ & 10.5 & 6 & 0.10 & 15.8 & -0.023 & 320 \\
\hline & & Mar. 2010 & $0.92(0.70-1.14)$ & $1.46 \pm 0.28$ & 9.23 & 6 & 0.13 & 15.3 & -0.026 & 320 \\
\hline & & Sep. 2010 & $1.23(0.74-1.73)$ & $0.91 \pm 0.33$ & 3.69 & 6 & 0.72 & 20.5 & -0.005 & 320 \\
\hline & Faisalabad & Sep. 2008 & $1.33(0.73-1.94)$ & $0.83 \pm 0.16$ & 6.18 & 6 & 0.40 & 22.2 & - & 320 \\
\hline & & Mar. 2009 & $1.20(0.66-1.70)$ & $0.82 \pm 0.24$ & 4.18 & 6 & 0.65 & 20 & -0.007 & 320 \\
\hline & & Sep. 2009 & $0.99(0.68-1.31)$ & $1.11 \pm 0.39$ & 9.71 & 6 & 0.14 & 16.5 & -0.021 & 320 \\
\hline & & Mar. 2010 & $0.94(0.65-1.22)$ & $1.13 \pm 0.42$ & 9.48 & 6 & 0.15 & 15.7 & -0.025 & 320 \\
\hline & & Sep. 2010 & $1.23(0.73-1.72)$ & $0.91 \pm 0.23$ & 4.31 & 6 & 0.64 & 20.5 & -0.006 & 320 \\
\hline & Sargodha & Sep. 2008 & $1.01(0.69-1.32)$ & $1.14 \pm 0.34$ & 6.36 & 6 & 0.38 & 16.8 & - & 320 \\
\hline & & Mar. 2009 & $0.90(0.61-1.20)$ & $1.04 \pm 0.22$ & 3.26 & 6 & 0.78 & 15 & -0.008 & 320 \\
\hline & & Sep. 2009 & $0.80(0.57-1.03)$ & $1.18 \pm 0.52$ & 3.54 & 6 & 0.74 & 13.2 & -0.017 & 320 \\
\hline & & Mar. 2010 & $0.92(0.58-1.26)$ & $0.92 \pm 0.24$ & 6.83 & 6 & 0.34 & 15.3 & -0.007 & 320 \\
\hline & & Sep. 2010 & $1.10(0.72-1.47)$ & $1.06 \pm 0.42$ & 3.09 & 6 & 0.80 & 18.3 & 0.006 & 320 \\
\hline \multirow[t]{16}{*}{ Thiodicarb } & Lab-PK & & $0.03(0.01-0.11)$ & $2.28 \pm 0.63$ & 1.28 & 6 & 0.97 & 1 & - & 320 \\
\hline & Lahore & Sep. 2008 & $1.05(0.71-1.37)$ & $1.13 \pm 0.23$ & 8.29 & 6 & 0.22 & 35 & - & 320 \\
\hline & & Mar. 2009 & $0.88(0.61-1.15)$ & $1.12 \pm 0.32$ & 3.99 & 6 & 0.68 & 29.3 & -0.013 & 320 \\
\hline & & Sep. 2009 & $0.90(0.61-1.19)$ & $1.07 \pm 0.41$ & 4.49 & 6 & 0.61 & 30 & -0.011 & 320 \\
\hline & & Mar. 2010 & $0.71(0.51-0.89)$ & $1.28 \pm 0.25$ & 6.13 & 6 & 0.41 & 23.7 & -0.028 & 320 \\
\hline & & Sep. 2010 & $0.99(0.68-1.28)$ & $1.17 \pm 0.23$ & 1.75 & 6 & 0.94 & 33 & -0.004 & 320 \\
\hline & Faisalabad & Sep. 2008 & $1.10(0.71-1.49)$ & $1.01 \pm 0.28$ & 7.92 & 6 & 0.24 & 36.7 & - & 320 \\
\hline & & Mar. 2009 & $0.89(0.63-1.18)$ & $1.09 \pm 0.30$ & 5.02 & 6 & 0.54 & 29.7 & -0.015 & 320 \\
\hline & & Sep. 2009 & $0.97(0.62-1.32)$ & $0.97 \pm 0.20$ & 4.86 & 6 & 0.56 & 32.3 & -0.009 & 320 \\
\hline & & Mar. 2010 & $0.76(0.54-0.98)$ & $1.18 \pm 0.42$ & 8.75 & 6 & 0.19 & 25.3 & -0.027 & 320 \\
\hline & & Sep. 2010 & $1.07(0.71-1.43)$ & $1.06 \pm 0.23$ & 4.11 & 6 & 0.66 & 35.7 & -0.002 & 320 \\
\hline & Sargodha & Sep. 2008 & $0.85(0.52-1.19)$ & $0.86 \pm 0.17$ & 5.00 & 6 & 0.54 & 28 & - & 320 \\
\hline & & Mar. 2009 & $0.83(0.58-1.09)$ & $1.14 \pm 0.29$ & 1.85 & 6 & 0.93 & 27.7 & -0.002 & 320 \\
\hline & & Sep. 2009 & $0.94(0.63-1.25)$ & $1.21 \pm 0.33$ & 4.41 & 6 & 0.62 & 31.2 & 0.007 & 320 \\
\hline & & Mar. 2010 & $0.71(0.53-0.90)$ & $1.31 \pm 0.44$ & 6.35 & 6 & 0.39 & 23.7 & -0.013 & 320 \\
\hline & & Sep. 2010 & $1.03(0.71-1.33)$ & $1.17 \pm 0.22$ & 3.74 & 6 & 0.71 & 34 & 0.014 & 320 \\
\hline \multirow[t]{16}{*}{ Indoxacarb } & Lab-PK & & $0.022(0.014-0.044)$ & $2.19 \pm 0.46$ & 1.73 & 6 & 0.94 & 1 & - & 320 \\
\hline & Lahore & Sep. 2008 & $0.91(0.59-1.21)$ & $1.02 \pm 0.24$ & 3.62 & 6 & 0.73 & 41.4 & _ & 320 \\
\hline & & Mar. 2009 & $1.29(0.76-1.83)$ & $0.90 \pm 0.17$ & 2.62 & 6 & 0.86 & 58.6 & 0.025 & 320 \\
\hline & & Sep. 2009 & $1.12(0.69-1.54)$ & $0.95 \pm 0.25$ & 4.76 & 6 & 0.57 & 50.9 & 0.015 & 320 \\
\hline & & Mar. 2010 & $1.18(0.83-1.52)$ & $1.28 \pm 0.34$ & 10.7 & 6 & 0.10 & 53.6 & 0.019 & 320 \\
\hline & & Sep. 2010 & $1.19(0.78-1.59)$ & $1.07 \pm 0.24$ & 4.58 & 6 & 0.60 & 54.1 & 0.019 & 320 \\
\hline & Faisalabad & Sep. 2008 & $1.21(0.64-1.76)$ & $0.78 \pm 0.16$ & 3.02 & 6 & 0.81 & 55 & - & 320 \\
\hline & & Mar. 2009 & $1.42(0.78-2.07)$ & $0.85 \pm 0.38$ & 4.00 & 6 & 0.68 & 64.5 & 0.012 & 320 \\
\hline & & Sep. 2009 & $1.87(0.66-2.31)$ & $0.63 \pm 0.24$ & 1.38 & 6 & 0.97 & 85 & 0.032 & 320 \\
\hline & & Mar. 2010 & $1.17(0.82-1.53)$ & $1.23 \pm 0.43$ & 9.78 & 6 & 0.13 & 53.1 & -0.002 & 320 \\
\hline & & Sep. 2010 & $2.19(0.37-4.00)$ & $0.51 \pm 0.11$ & 3.19 & 6 & 0.78 & 99.5 & 0.043 & 320 \\
\hline & Sargodha & Sep. 2008 & $1.62(0.56-2.69)$ & $0.59 \pm 0.18$ & 3.77 & 6 & 0.71 & 73.6 & - & 320 \\
\hline & & Mar. 2009 & $1.37(0.73-2.00)$ & $0.83 \pm 0.42$ & 1.98 & 5 & 0.85 & 62.3 & -0.012 & 280 \\
\hline & & Sep. 2009 & $1.59(0.78-2.38)$ & $0.80 \pm 0.23$ & 3.54 & 6 & 0.74 & 72.3 & -0.001 & 320 \\
\hline & & Mar. 2010 & $1.37(0.77-1.89)$ & $1.05 \pm 0.25$ & 12.0 & 6 & 0.07 & 62.2 & -0.012 & 320 \\
\hline & & Sep. 2010 & $1.96(0.60-3.33)$ & $0.60 \pm 0.18$ & 3.42 & 6 & 0.75 & 89.1 & 0.014 & 320 \\
\hline \multirow[t]{3}{*}{ Emamectin benzoate } & Lab-PK & & $0.09(0.04-0.14)$ & $2.35 \pm 0.98$ & 2.85 & 6 & 0.83 & 1 & - & 320 \\
\hline & Lahore & Sep. 2008 & $1.39(0.91-1.86)$ & $1.16 \pm 0.26$ & 9.23 & 6 & 0.16 & 15.4 & - & 320 \\
\hline & & Mar. 2009 & $1.65(0.59-2.70)$ & $0.64 \pm 0.22$ & 0.41 & 4 & 0.98 & 18.3 & 0.013 & 240 \\
\hline
\end{tabular}


Table 2 Toxicity of carbamates and new insecticides against field populations of Ae. albopictus (Continued)

\begin{tabular}{|c|c|c|c|c|c|c|c|c|c|c|}
\hline & & Sep. 2009 & $2.07(0.60-3.54)$ & $0.50 \pm 0.12$ & 7.02 & 6 & 0.32 & 23 & 0.029 & 320 \\
\hline & & Mar. 2010 & $1.61(0.92-2.29)$ & $1.00 \pm 0.27$ & 9.62 & 6 & 0.14 & 17.9 & 0.011 & 320 \\
\hline & & Sep. 2010 & $2.45(1.35-4.26)$ & $0.52 \pm 0.26$ & 2.66 & 6 & 0.85 & 27.2 & 0.041 & 320 \\
\hline & Faisalabad & Sep. 2008 & $1.35(0.87-1.82)$ & $1.10 \pm 0.25$ & 7.78 & 6 & 0.26 & 15 & - & 320 \\
\hline & & Mar. 2009 & $1.97(1.21-3.32)$ & $0.61 \pm 0.24$ & 4.78 & 6 & 0.57 & 21.9 & 0.027 & 320 \\
\hline & & Sep. 2009 & $1.47(0.91-2.02)$ & $1.07 \pm 0.26$ & 10.9 & 6 & 0.91 & 16.3 & 0.006 & 320 \\
\hline & & Mar. 2010 & $2.00(1.12-3.17)$ & $0.76 \pm 0.18$ & 4.53 & 6 & 0.61 & 22.2 & 0.028 & 320 \\
\hline & & Sep. 2010 & $1.89(0.68-2.50)$ & $0.65 \pm 0.23$ & 1.39 & 6 & 0.98 & 21 & 0.024 & 320 \\
\hline & Sargodha & Sep. 2008 & $1.14(0.85-1.46)$ & $1.31 \pm 0.25$ & 7.11 & 6 & 0.31 & 12.7 & - & 320 \\
\hline & & Mar. 2009 & $1.26(0.89-1.63)$ & $1.33 \pm 0.26$ & 4.32 & 6 & 0.63 & 14 & 0.007 & 320 \\
\hline & & Sep. 2009 & $1.67(0.88-2.59)$ & $0.73 \pm 0.24$ & 5.96 & 6 & 0.43 & 18.6 & 0.028 & 320 \\
\hline & & Mar. 2010 & $1.70(0.86-2.54)$ & $0.86 \pm 0.30$ & 7.47 & 5 & 0.19 & 18.9 & 0.029 & 320 \\
\hline & & Sep. 2010 & $1.30(0.91-1.75)$ & $1.10 \pm 0.22$ & 3.74 & 6 & 0.71 & 14.4 & 0.010 & 320 \\
\hline \multirow[t]{16}{*}{ Spinosad } & Lab-PK & & $0.019(0.02-0.13)$ & $2.71 \pm 0.44$ & 2.92 & 6 & 0.82 & 1 & - & 320 \\
\hline & Lahore & Sep. 2008 & $0.53(0.40-0.66)$ & $1.45 \pm 0.32$ & 3.22 & 6 & 0.78 & 27.9 & - & 320 \\
\hline & & Mar. 2009 & $0.51(0.39-0.61)$ & $1.78 \pm 0.24$ & 3.02 & 6 & 0.81 & 26.8 & -0.003 & 320 \\
\hline & & Sep. 2009 & $0.48(0.38-0.57)$ & $1.09 \pm 0.26$ & 9.25 & 6 & 0.16 & 25.3 & -0.007 & 320 \\
\hline & & Mar. 2010 & $0.57(0.44-0.69)$ & $1.56 \pm 0.42$ & 7.74 & 6 & 0.26 & 30 & 0.005 & 320 \\
\hline & & Sep. 2010 & $0.59(0.43-0.72)$ & $1.55 \pm 0.42$ & 10.2 & 6 & 0.13 & 31.1 & 0.008 & 320 \\
\hline & Faisalabad & Sep. 2008 & $0.63(0.46-0.81)$ & $1.27 \pm 0.23$ & 8.32 & 6 & 0.22 & 33.2 & - & 320 \\
\hline & & Mar. 2009 & $0.73(0.55-0.91)$ & $1.43 \pm 0.38$ & 5.18 & 6 & 0.52 & 38.4 & 0.011 & 320 \\
\hline & & Sep. 2009 & $0.50(0.38-0.62)$ & $1.52 \pm 0.24$ & 10.1 & 6 & 0.12 & 26.3 & -0.012 & 320 \\
\hline & & Mar. 2010 & $0.43(0.30-0.56)$ & $1.29 \pm 0.23$ & 12.2 & 6 & 0.06 & 22.6 & -0.028 & 320 \\
\hline & & Sep. 2010 & $0.95(0.70-1.21)$ & $1.26 \pm 0.43$ & 2.12 & 6 & 0.91 & 50 & 0.030 & 320 \\
\hline & Sargodha & Sep. 2008 & $0.56(0.41-0.71)$ & $1.33 \pm 0.22$ & 7.79 & 6 & 0.25 & 29.5 & - & 320 \\
\hline & & Mar. 2009 & $0.73(0.54-0.93)$ & $1.29 \pm 0.22$ & 4.91 & 6 & 0.56 & 38.4 & 0.019 & 320 \\
\hline & & Sep. 2009 & $0.52(0.39-0.64)$ & $1.50 \pm 0.36$ & 10.0 & 6 & 0.16 & 27.4 & -0.005 & 320 \\
\hline & & Mar. 2010 & $0.43(0.29-0.56)$ & $1.22 \pm 0.23$ & 9.93 & 6 & 0.13 & 22.6 & -0.019 & 320 \\
\hline & & Sep. 2010 & $0.85(0.62-1.07)$ & $1.44 \pm 0.56$ & 3.16 & 6 & 0.79 & 44.7 & 0.030 & 320 \\
\hline
\end{tabular}

*Resistance ratio $=\mathrm{LC}_{50}$ field population/ $\mathrm{LC}_{50}$ of susceptible strain

${ }^{* *}$ Rate of increase or decrease in resistance ${ }^{* * *}$ Number of larvae tested in a bioassay

moderately resistant (14-27 fold) compared with Lab-PK (Table 2). The lowest level was found in populations from Sargodha in March 2009. Moderate to high levels of resistance were observed in populations tested for spinosad (23-50 fold, Table 2) compared with Lab-PK. One population from Lahore, three populations from Faisalabad and two from Sargodha had high levels of resistance while the remaining populations were moderately resistant (Table 2). The slopes of regression lines of all the field populations were similar $(\mathrm{P}>0.05)$.

\section{Pair wise correlations between $\log \mathrm{LC}_{50 \text { s }}$ of different insecticides}

Correlation between emamectin benzoate and spinosad in the new chemicals group was non- significant $(\mathrm{P}>$ 0.05); however, resistance to emamectin benzoate was significantly $(\mathrm{P}<0.05)$ correlated with profenofos and lambdacyhalothrin, but no significant correlation was found between emamectin benzoate and chlorpyrifos, triazofos, cypermethrin, deltamethrin, methomyl, thiodicarb and indoxacarb (Table 3). In contrast, spinosad had significant correlation with thiodicarb and indoxacarb but no correlation with the remaining insecticides. Indoxacarb had significant correlation with chlorpyrifos only. The $\mathrm{LC}_{50}$ values of the insecticides of carbamate group had highly significant $(\mathrm{P}<$ 0.01 ) correlation within the group. However, thiodicarb had also a significant correlation with deltamethrin, and methomyl with cypermethrin and deltamethrin. Within the pyrethriod group, deltamethrin and cypermethrin had significant correlation $(\mathrm{P}<0.05)$. All the pyrethroids had a significant correlation with profenofos. Moreover, insecticides in organophosphate group had non significant $(\mathrm{P}>0.05)$ correlation with each other (Table 3).

\section{Stability of resistance across 3 years}

From 2008 to 2010, resistance of Ae. albopictus to all tested insecticides remained the same. There was no indication of significant change $(\mathrm{P}>0.05)$ in the rate of 
Table 3 Pairwise comparisons of correlation coefficient between $\log \mathrm{LC}_{50}\left(\mu \mathrm{g} \mathrm{mL}^{-1}\right.$ ) values of the tested insecticides in field populations of Ae. albopictus

\begin{tabular}{|c|c|c|c|c|c|c|c|c|c|c|}
\hline Insecticide & Chlorpyrifos & Profenofos & Triazofos & Cypermethrin & Deltamethrin & Lambdacyhalothrin & Methomyl & Thiodicarb & Indoxacarb & Emamectin \\
\hline \multicolumn{11}{|l|}{ Chlorpyrifos } \\
\hline Profenofos & $0.33^{\text {ns }}$ & & & & & & & & & \\
\hline Triazofos & $-0.37^{\mathrm{ns}}$ & $0.08^{\mathrm{ns}}$ & & & & & & & & \\
\hline Cypermethrin & $0.28^{\mathrm{ns}}$ & $0.69^{<0.01}$ & $0.29^{\text {ns }}$ & & & & & & & \\
\hline Deltamethrin & $-0.07^{\mathrm{ns}}$ & $0.52^{<0.05}$ & $0.37^{\mathrm{ns}}$ & $0.52^{<0.05}$ & & & & & & \\
\hline Lambdacyhalothrin & $0.58^{<0.05}$ & $0.47^{<0.05}$ & $-0.36^{\text {ns }}$ & $0.37^{\mathrm{ns}}$ & $0.39^{\mathrm{ns}}$ & & & & & \\
\hline Methomyl & $-0.02^{\text {ns }}$ & $0.04^{\mathrm{ns}}$ & $0.27^{\mathrm{ns}}$ & $0.47^{<0.05}$ & $0.67^{<0.01}$ & $0.38^{\text {ns }}$ & & & & \\
\hline Thiodicarb & $-0.27^{\mathrm{ns}}$ & $-0.01^{\mathrm{ns}}$ & $0.28^{\mathrm{ns}}$ & $0.27^{\mathrm{ns}}$ & $0.50^{<0.05}$ & $0.24^{\mathrm{ns}}$ & $0.71^{<0.01}$ & & & \\
\hline Indoxacarb & $-0.48^{<0.05}$ & $-0.08^{\text {ns }}$ & $0.54^{\mathrm{ns}}$ & $-0.09^{\text {ns }}$ & $-0.04^{\text {ns }}$ & $-0.40^{\text {ns }}$ & $-0.06^{\mathrm{ns}}$ & $0.30^{\mathrm{ns}}$ & & \\
\hline Emamectin benzoate & $0.18^{\text {ns }}$ & $0.52^{<0.05}$ & $-0.41^{\text {ns }}$ & $0.14^{\mathrm{ns}}$ & $0.31^{\mathrm{ns}}$ & $0.51^{<0.05}$ & $0.08^{\text {ns }}$ & $-0.07^{\text {ns }}$ & $-0.20^{\mathrm{ns}}$ & \\
\hline Spinosad & $-0.34^{\mathrm{ns}}$ & $-0.22^{\mathrm{ns}}$ & $0.35^{\mathrm{ns}}$ & $-0.07^{\mathrm{ns}}$ & $0.16^{\mathrm{ns}}$ & $-0.19^{\text {ns }}$ & $0.41^{\mathrm{ns}}$ & $0.49^{<0.05}$ & $0.62^{<0.05}$ & $-0.12^{\text {ns }}$ \\
\hline
\end{tabular}

(Superscripts indicate the significance of correlation) 
increase or decrease in resistance to the insecticides tested (Table 1,2).

\section{Discussion}

Pakistan has a long history of insecticide resistance problems in cotton pests like Helicoverpa armigera, Bemisia tabaci, Aphis gosypii [25], Spodoptera litura [24] and even in the generalist predator, the green lacewing, Chrysoperla carnea [16]. Here we have shown a strong indication of resistance in Ae. albopictus collected from areas of high agrochemical use compared to the Lab-PK population with zero agrochemicals exposure. The present experiments were carried out to evaluate the resistance of three insecticides from each of organophosphate, pyrethroid, new chemicals and two from carbamates. These insecticides have intensively been used to combat various cotton pests in Pakistan for the last two decades [26]. The experiments were conducted for 3 consecutive years (2008-2010), and the results of bioassays showed varying degrees of resistance in field populations. Resistance in Ae. albopictus to chlorpyrifos was generally very high while moderate to high levels of resistance were found with remaining insecticides. Insect populations should be considered susceptible if a resistance ratio of 10 is exhibited [27], however, none of the field populations was found to have resistance ratio 10 or below 10 . The present studies suggest that Ae. albopictus might have evolved resistance to agrochemicals due to possible cross resistance mechanisms among various agrochemicals.

Pair wise correlation coefficient comparisons of log $\mathrm{LC}_{50}$ values of insecticides for field populations showed positive correlations among most of the insecticides (Table 3), suggesting a cross resistance mechanism. The presence of two divergent patterns of correlation within agrochemicals indicates that more than one mechanism of resistance exists for imparting resistance to agrochemicals in Ae. albopictus. High levels of resistance to most of the insecticides might be due to multiple resistance mechanism [17]. The mixing of new chemicals with conventional insecticides could be responsible for creating multiple resistance problems, which has been reported in Spodoptera sp. from other parts of the world [28]. Owing to the common practice of mixing new compounds with conventional insecticides to control cotton pests in Pakistan [17] it would be untimely to conclude that cross resistance exists in Ae. albopictus against these agrochemicals. However further studies are required to confirm whether the cross resistance between insecticides exists by selecting Ae. albopictus population in the laboratory with representative insecticides.

In the present investigations Ae. albopictus larvae were found resistant to all classes of tested chemicals which could be due to one or more than one resistance mechanisms involved. The resistance of Aedes larvae to pyrethroids and organophosphates has also been reported from other parts of the world [1]. Insecticide resistance mechanism in mosquitoes has extensively been studied in the past [29]. The resistance to pyrethroids in mosquitoes is mainly conferred by two mechanisms: (a) mutation in the voltage-gated sodium channel or (b) by elevated levels of monooxygenases $[30,31]$. In contrast, over expression of esterases by gene amplification or mutation provides considerable organophosphate (and to some extent carbamate) resistance in mosquitoes, and has been considered an evolutionary response to selection by organophosphates and carbamates [31]. Monooxygenases play a minor role in organophosphate resistance, and little, if any, in resistance to carbamates [32].

In the current study, rate of increase or decrease of resistance to insecticides in the field population of $A e$. albopictus was minimal, suggesting that resistance was stable in the populations collected from various locations. The stability of resistance in field collected samples conferred at least one justification. The resistance might have been near fixation, leading to a very slow increase in heterozygosity owing to combination of ecological, biological and/or biochemical (reduced detoxification capacity) factors [16,33].

Though the Aedes larvae have not been directly exposed to agrochemicals applied for the cotton pest management, the results of present study clearly showed the field evolved resistance to agrochemicals. The use of insecticides in agricultural crop protection could indeed affects resistance development in disease vectors $[13,34,35]$. The cotton crop in Pakistan is usually sprayed with more than 34 insecticides of different chemical classes, including premix and tank mix products [26]. These chemicals are mainly organophosphates, carbamates, pyrethroids, neonicotinoids and new chemistries and are used either as a single formulation or in combinations of two or three insecticides of different classes, the final aim being to generate a synergistic effect of insecticides for a better pest management. After pesticide treatments in agricultural crops, insecticides residues drift into mosquito breeding sites [9]. These residues have lethal effects on larvae of some populations of mosquitoes whereas they exercise a selective pressure on other populations, leading to the emergence of resistant populations [10]. Moreover, considerable agrochemical-insect contact could occur during mosquito flights between breeding habitats and blood sources and resting places, potentially increasing selection pressure to insecticides [9]. Moreover insecticides used in public health programs against disease vectors are similar to those used for years in agriculture 
[10]. Several hypotheses concerning the resistance in disease vectors have emerged e.g. some researchers incriminate pesticide use in cotton and rice fields as the main source of resistance selection in several species of mosquitoes in rural environments [35-39]. One important threat that may compromise future control efforts is the potential for cross resistance between organophosphates and pyrethroids. Resistance to pyrethroids has generally been associated with cross resistance to DDT [40], however, an esterase-based resistance mechanism in An. albimanus conferred cross resistance between pyrethroid (deltamethrin) and organophosphate (fenithrothion) [41]. In areas where organophosphate has extensively been used for agricultural pest control, such cross resistance may pose a potential threat to future control of the dengue vector.

\section{Conclusions}

We now plan to look for pesticide residues in mosquito breeding sites to confirm the actual involvement of agrochemicals in the selection of resistance in Ae. albopictus. In conclusion, it is recommended that regular resistance surveillance should first be focused in areas where dengue fever transmission and intensive chemical agricultural pest control coincide, because these areas are more prone to develop insecticide resistance in $A e$. albopictus. Continuous resistance monitoring will also result to identify the efficacy of compounds for dengue control and to facilitate selection of compounds with the greatest promise to minimize dengue infections. Moreover, public awareness about dengue, cooperation with public health campaigns to eliminate larval Aedes breeding habitats and Insecticide Resistance Management in combination with Integrated Pest and Vector Management are recommended strategies for controlling dengue vectors and to reduce risks to humans as well as environmental health.

\section{Acknowledgements}

We are greatly thankful to Higher Education Commission (HEC) Pakistan for providing funds to carry out this experiment.

\section{Author details}

'Department of Entomology, University College of Agriculture, Bahauddin Zakariya University, Multan, Pakistan. ${ }^{2}$ Department of Agri-Entomology, University of Agriculture, Faisalabad, Pakistan. ${ }^{3}$ Department of Biology, Islamia University of Bahawalpur, Pakistan. ${ }^{4}$ Department of Zoology, South Valley University, Aswan, Egypt.

\section{Authors' contributions}

HAAK conceived, designed, performed the study, analyzed the data and wrote the manuscript. WA supervised the study and helped draft the manuscript. KS helped in mosquito collections and bioassays and EASS helped draft the manuscript. All authors approved the final version of the manuscript.

\section{Competing interests}

The authors declare that they have no competing interests.
Received: 13 June 2011 Accepted: 22 July 2011 Published: 22 July 2011

\section{References}

1. Ponlawat J, Scott G, Harrington LC: Insecticide susceptibility of Aedes aegypti and Aedes albopictus across Thailand. J Med Entomol 2005, 42:821-825.

2. Jacobs M: Dengue: emergence as a global public health problem and prospects for control. Trans R Soc Trop Med Hyg 2000, 94:7-8.

3. Din S, Akram W, Khan HAA, Hussain A, Hafeez F: Citrus waste-derived essential oils: Alternative larvicides for dengue fever mosquito, Aedes albopictus (Skuse) (Culicidae:Diptera). Pak J Zool 2011, 43:367-372.

4. Akram W, Khan HAA, Hafeez F, Bilal H, Kim YK, Lee JJ: Potential of citrus seed extracts against dengue fever mosquito, Aedes albopictus (Skuse) (Culicidae: Diptera). Pak J Bot 2010, 42:3343-3348.

5. Denolm I, Elliot M: Bibliographic memoirs of fellows of the royal society. (Roman): Mieczyslaw Sawicki 1995, 397-417.

6. Van Emden H: Pest Control. Edward Arnold Publishers, London, United Kingdom; 1989.

7. Anonymous: Arthropod pesticide resistance database. 2007 [http://www. pesticideresistance.org/search/1].

8. Mota-Sanchez D, Bills PS, Whalon ME: Arthropod resistance to pesticides: Status and overview. In: Pesticides in agriculture and the environment. Edited by: WB Wheeler. New York, Marcel Dekker Inc.; 2002:241-272.

9. Overgaard HJ, Sandve SR, Suwonkerd W: Evidence of anopheline mosquito resistance to agrochemicals in northern Thailand. Southeast Asian J Trop Med Pub Health 2005, 36:148-153.

10. Akogbeto M, Jouaka RFD, Kindé-Gazard DA: Screening of pesticide residues in soil and water samples from agricultural settings. Mal J 2006, 5:22-31.

11. Georghiou GP, Ariaratnam V, Breeland SG: Anopheles albimanus: development of carbamate and organophosphorus resistance in nature. Bull World Health Organ 1971, 46:551-554.

12. Chapin G, Wasserstrom R: Agricultural production and malaria resurgence in Central America and India. Nature 1981, 293:181-185.

13. Brogdon WG, Beach RF, Stewart JM, Castanza L: Microplate assay analysis of the distribution of organophosphate and carbamate resistance in Guatemalan Anopheles albimanus. Bull. World Health Organ 1988, 66:339-346.

14. Diabate A, Baldet $T$, Chandre $F$, et al: The role of agricultural use of insecticides in resistance to pyrethroids in Anopheles gambiae in Burkina Faso. Am J Trop Med Hyg 2002, 67:617-22.

15. Sharma VP: Re-emergence of malaria in India. Indian J Med Res 1996, 103:26-45

16. Pathan AK, Sayyed AH, Aslam M, Razaq M, Jilani G, Saleem MA: Evidence of field evolved resistance to organophosphates and pyrethroids in Chrysoperla carnea (Neuroptera: Chrysopidae). J Econ Entomol 2008, 101:1676-1684.

17. Ahmad M, Sayyed AH, Saleem MA, Ahmad M: Evidence for field evolved resistance to newer insecticides in Spodoptera litura (Lepidoptera: Noctuidae) from Pakistan. Crop Prot 2008, 27:1367-1372.

18. Bisset J, Rodriguez M, Soca A, Pasteur N, Raymond M: Cross resistance to pyrethroid and organophosphorus insecticides in the southern house mosquito from Cuba. J Med Entomol 1997, 34:244-246.

19. Abbott SW: A method of computing the effectiveness of an insecticide. J Econ Entomol 1925, 18:265-267.

20. Finney DJ: Probit Analysis. Cambridge University Press, Cambridge, UK; 3 1971.

21. Minitab Inc.: MINITAB statistical software, releases 15 for Windows, State College, PA. 2009.

22. Litchfield JT, Wilcoxon F: A simplified method of evaluating dose effect experiments. J Pharmacol Exp Ther 1949, 99:99-103.

23. Analytical Software, Statistix version 8.1: User's manual. Analytical Software, Tallahassee, Florida; 2005.

24. Ahmad M, Arif I, Ahmad M: Occurrence of insecticide resistance in field populations of Spodoptera litura (Lepidoptera: Noctuidae) in Pakistan. Crop Prot 2007, 26:807-811.

25. Ahmad Ml, Arif I, Denholm : High resistance of field populations of the cotton aphid Aphis gossypii Glover (Homoptera: Aphididae) to pyrethroid insecticides in Pakistan. J Econ Entomol 2003, 96:875-878.

26. Saleem MA, Ahmad A, Ahmad M, Aslam M, Sayyed AH: Resistance to Selected organochlorine, organophosphate, carbamates and pyrethroid, 
in Spodoptera litura (Lepidoptera:Noctuidae) from Pakistan. J Econ Entomol 2008, 101:1667-1675.

27. Valles $S M$, Koehler PG, Brenner RJ: Antagonism of fipronil toxicity by piperonyl butoxide and S,S,S-tributyl phosphorotrithioate in the German cockroach (Dictyoptera: Blattellidae). J Econ Entomol 1997, 90:1254-1258.

28. Yu SJ, Nguyen SN, Abo-Elghar GE: Biochemical characteristics of insecticide resistance in the fall army worm, Spodoptera frugiperda (J.E. Smith). Pestic Biochem Physiol 2003, 77:1-11.

29. Hemingway J, Ranson $\mathrm{H}$ : Insecticides resistance in insect vectors of human disease. Annu Rev Entomol 2000, 45:371-391.

30. Liu N, Xu Q, Zhu F, Zhang L: Pyrethroid resistance in mosquitoes. Insect Sci 2006, 13:159-166.

31. Hemingway J, Hawkes NJ, McCarroll L, Ranson H: The molecular basis of insecticide resistance in mosquitoes. Insect Biochem Mol Biol 2004, 34:653-665.

32. Nauen $R$ : Insecticide resistance in disease vectors of public health importance. Pest Manag Sci 2007, 63:628-633.

33. Roush RT, Hoy CW, Ferro DN, Tingey WM: Insecticide resistance in the Colorado potato beetle (Coleoptera, Chrysomelidae) influence of croprotation and insecticide use. J Econ Entomol 1990, 83:315-319.

34. Hemingway J, Jayawardena KG, Herath PR: Pesticide resistance mechanisms produced by field selection pressures on Anopheles nigerrimus and A. culicifacies in Sri Lanka. Bull World Health Organ 1986, 64:753-758,

35. Diabate A, Baldet T, Chandre F, Guiguemde TR, Guillet P, Hemingway J, Hougard JM: First report of the $k d r$ mutation in Anopheles gambiae from Burkina Faso, West Africa. Parasitologia 2002, 44:157-158.

36. Georghiou GP, Lagunes-Tejada A: Cases of resistance in Insecta. The Occurrence of resistance to pesticides in arthropods. Food and Agriculture Organisation of the United Nations: Rome; 1991.

37. Chandre F, Darriet F, Manga L, Akogbéto M, Faye O, Mouchet J, Guillet P: Status of pyrethroid resistance in Anopheles gambiae. Bull World Health Organ 1999, 77:230-234.

38. N'guessan R, Darriet F, Guillet P, Carnevale P, Traoré-Lamizana M, Corbel V, Koffi AA, Chandre F: Resistance to carbosulfan in field populations of Anopheles gambiae from Côte d' Ivoire basedon reduced sensitivity of acetylcholinesterase. Med Vet Entomol 2003, 17:19-25.

39. Kamgang B, Marcombe $S$, Chander $F$, et al: Insecticide susceptibility of Aedes Aegypti and Aedes albopictus in Central Africa. Parasites Vectors 2011, 4:79.

40. Ranson H, Jensen B, Vulule JM, Wang X, Hemingway J, Collins FH: Identification of a point mutation in the voltage-gated sodium channel gene of Kenyan Anopheles gambiae associated with resistance to DDT and pyrethroids. Insect Mol Biol 2000, 9:491-497.

41. Brogdon WG, Barber AM: Fenitrothion-deltamethrin cross-resistance conferred by esterases in Guatemalan Anopheles albimanus. Pestic Biochem Physiol 1990, 3:130-139.

\section{Submit your next manuscript to BioMed Central and take full advantage of:}

- Convenient online submission

- Thorough peer review

- No space constraints or color figure charges

- Immediate publication on acceptance

- Inclusion in PubMed, CAS, Scopus and Google Scholar

- Research which is freely available for redistribution 